\title{
BIFURCATION IN HYDRODYNAMIC MODELS OF
}

STELLAR PULSATION

\author{
T. AIKAWA \\ Tohoku Gakuin University, Faculty of Liberal Arts, \\ Izumi-ku, Sendai 981-31, Japan
}

\begin{abstract}
Phenomena of bifurcation in hydrodynamic stellar models of radial pulsation are reviewed. By changing control parameters of models, we can see qualitatively different pulsation behaviors in hydrodynamic models with transitions due to various types of bifurcation.

In weakly dissipative models (classical Cepheids), the bifurcation is induced by modal resonances. Two ty pes of the modal resonances found in models are discussed: The higherharmonic resonances of the second overtone mode in the fundamental mode pulsator and of the fourth overtone mode in the first overtone pulsator are relevant to observations. The subharmonic resonance between the fundamental and first overtone modes is confirmed in classical Cepheid models.

In strongly dissipative models (less-massive supergiant stars), the bifurcation of nonlinear pulsation is induced by the hydrodynamics of ionization zones as well as modal resonances. The sequence of the bifurcation sometimes leads to chaotic behaviors in nonlinear pulsation. The transition routes from regular to the chaotic pulsations found in models are discussed with respect to the theory of chaos in simple dynamical systems: The cascade of period-doubling bifurcation is confirmed to cause chaotic pulsation in $\mathrm{W}$ Virginis models. For models of higher luminosity, the tangent bifurcation is found to lead intermittent chaos.

Finally, hydrodynamic models for chaotic pulsation with small amplitudes observed in the post-AGB stars are briefly discussed.
\end{abstract}

\section{Introduction}

We can guess stellar parameters and physical properties of pulsating stars with comparison of observational data with corresponding outputs of hydrodynamic models. This is an important purpose for hydrodynamic modeling of pulsating stars. In linear models, only pulsation periods are used for these comparisons, while pulsation periods give us rich information in multimode pulsators, particularly in nonradial pulsators (Saio, 1992). In nonlinear models, however, we can use the time variations of magnitude and velocity, although observations for these quantities are time consuming and the outputs for these quantities from hydrodynamic models depend on parameters for numerical treatments and assumptions used for simplifying hydrodynamic models.

The pulsation behaviors in hydrodynamic models are changed qualitatively with transitions due to bifurcation of nonlinear pulsation. In my review, we discuss these transitions in hydrodynamic models of radial pulsation for classical Cepheids and less-massive supergiant stars. Finally we discuss chaotic pulsations with small amplitudes in hydrodynamic models for the post-Asymptotic Giant Branch Stars. 


\section{Bifurcation in Weakly Dissipative Models (Cepheid Models)}

Bifurcation in nonlinear pulsations of weakly dissipative models (Cepheid models) is induced by modal resonances. There are two types of resonance: higher harmonic resonance and subharmonic resonance.

\subsection{Higher Harmonic Resonanices}

Modal resonance with the damped second overtone in the fundamental pulsator is well-known for a mechanism of the features in 10 days bump Cepheids (Simon and Schmidt. 1976). The higher harmonic component of the fundamental pulsation is induced in non-linear pulsation by this resonance. Many hydrodynamic models have been built to reproduce the feature of bump (Simon and Davis, 1983; Takeuti et al., 1983; Aikawa, 1987; Carson and Stothers, 1988, Fadeyev, 1992). Recently, Buchler et al. (1990) have made an intensive survey of hydrodynamic models for bump features. They have reproduced the Hertzsprung progression of velocity curves observed in bump Cepheids nicely.

Moskalik and Buchler (1990) have reported that other higher harmonic resonances of damped modes in the fundamental pulsator with $P_{f}=m P_{n}$ (where $P_{f}$ and $P_{n}$ are periods of the fundamental and $n$-th overtone modes, and $m$ is an integer) are realized in their Cepheid models.

According to analytical theories for these resonances (e.g., Takeuti and Aikawa, 1981), bifurcation of unstable pulsation from stable pulsation with the bump feature is predicted near the center of the resonance. Thus, we include this type resonance as a phenomenon of bifurcation.

\subsection{Subharmonic Resonances}

The Floquet stability analysis (e.g., Iooss and Joseph, 1980) of limit cycles in Cepheid models has been performed by Moskalik and Buchler (1990). They confirm effects of subharmonic resonances between the fundamental mode and overtone modes with the condition $P_{f}=m / 2 P_{n}$ where $m$ is an odd integer, for instance 3 or 5 . In particular, they have found that subharmonic bifurcation with period doubling is induced by the subharmonic resonance between the fundamental and first overtone modes with the condition $P_{f}=$ $3 / 2 P_{1}$, when both the modes have unstable limit cycles. Instead of limit cycles, the model has stable periodic oscillation with a period of twice the fundamental period. This type of the subharmonic resonance is found in other Cepheid models (Moskalik and Buchler, 1991).

\subsection{First Overtone Pilsator}

Antonello, Poretti and Redzzi (1990) have suggested that s-Cepheids are first overtone pulsators, and moreover the progression of light curves which is demonstrated as a trend in Fourier components obtained for light curves. 


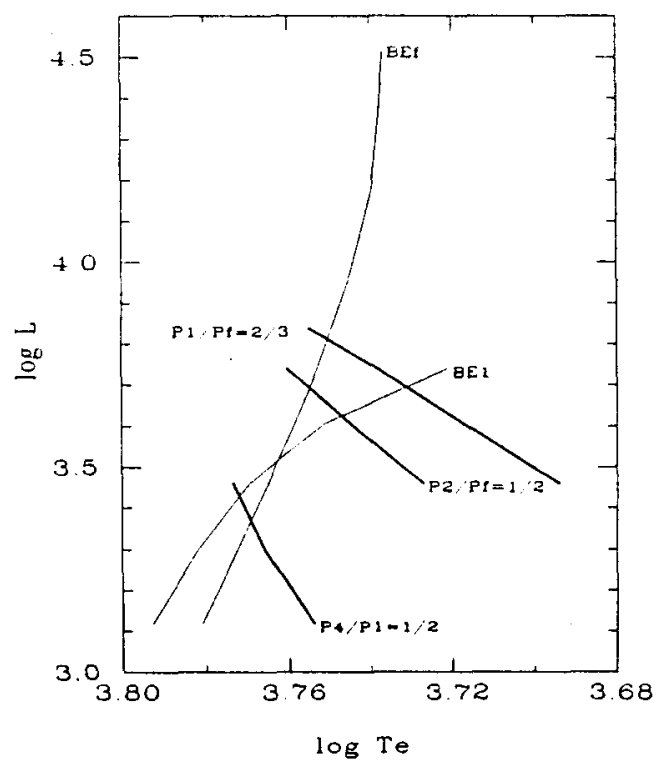

Fig. 1. Resonance lines in the HR diagram. Resonance lines for the fundamental and first overtone pulsators are drawn with labels of resonance conditions. The blue edges of the instability strip for the fundamental (BEf) and the first overtone mode (BE1) are also plotted.

Recently Antonello (1991) has suggested that modal resonance of the first overtone pulsator with the fourth overtone mode for the mechanism of the progression in s-Cepheids. We examine this possibility. Fig. 1 displays the location of the resonance in the HR diagram using the Mass-Luminosity relation adapted from Chiosi (1990) and the augmented metal opacities (Simon, 1982). Other resonance lines for the fundamental pulsator are also displayed in the figure. The factor for the augmented metal opacities is set so that the resonance for dump cepheids with $P_{f}=2 P_{2}$ is realized in the fundamental pulsator with periods around 10 days.

The non-linear simulations for the first overtone pulsator with the suggested resonance are performed by TGRID hydro code (Simon and Aikawa, 1986) for models with $M=4.0 M_{\odot}, L=1316 L_{\odot}$, and the effective temperatures are chosen for models to cover the resonance.

Fig. 2 demonstrates light curves of the models as a function of effective temperature and they show a systematic trend, as shown in Fig. 3 for $\phi_{21}$ and $R_{21}$ of the Fourier decomposition (see Simon and Lee, 1981 for definition of $\phi_{21}$ and $R_{21}$ ). We conclude that the higher harmonic resonance in the first overtone pulsator can make the features of the resonance in light curves.

This resonance with the damped fourth overtone mode in the first overtone pulsator is interesting also to the problem of double-mode Cepheids 


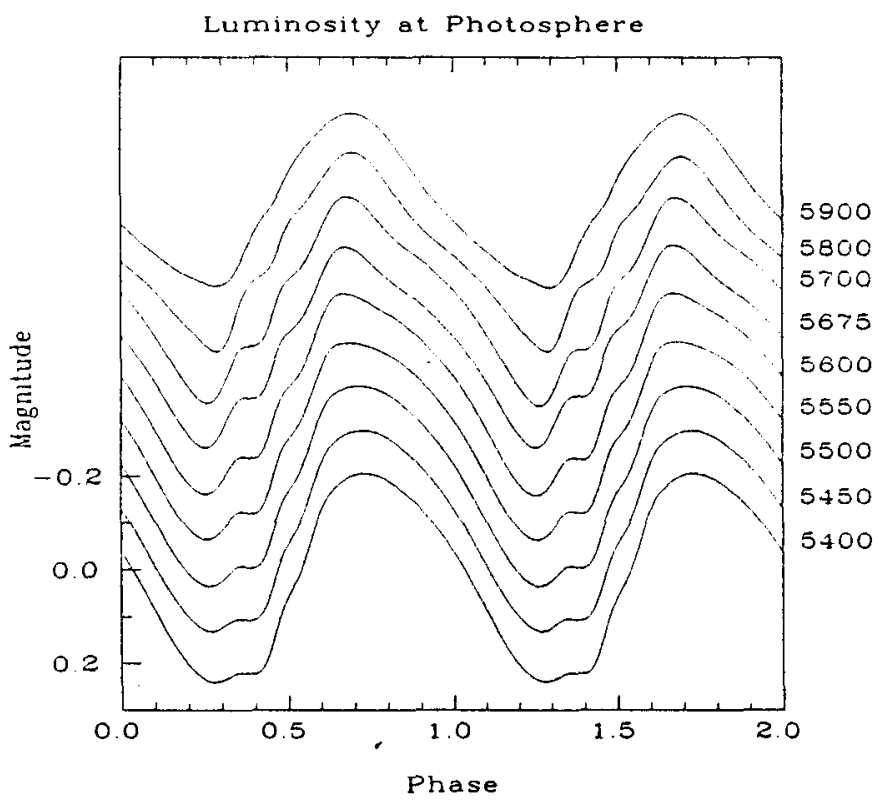

Fig. 2. Light curves for the limit cycle models of the first overtone pulsators in the resonance. The effective temperature of models is labeled for each curve which is shifted vertically.

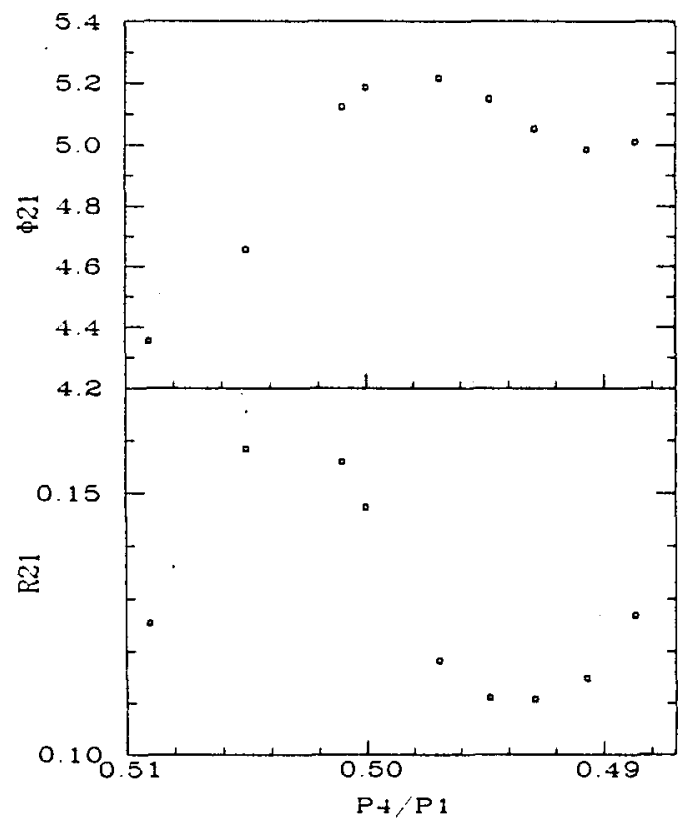

Fig. 3. The Fourier phases $\phi_{21}$ and Fourier amplitude ratios $R_{21}$ versus linear period ratio, $P_{4} / P_{1}$. 
(Balona, 1985) which have similar periods with s-Cepheids. Buchler et al. (1990) found a Cepheid model which has unstable limit cycles for the fundamental and first overtone modes, and confirmed a persistent beat behavior. In their models, the fundamental mode is in a very close to the higher harmonic resonance of damped second overtone mode, and they have suggested that the resonance lowers the fundamental mode amplitude and leads to destabilization of the limit cycle. If this reasoning is correct. the present resonance also will work for destabilization of the first overtone limit cycle.

\section{Bifurcation in Strongly Dissipative Models}

(less massive supergiant stars)

Pulsations in strongly dissipative models (less-massive supergiant stars) are characterized by appearance of irregular behaviors (e.g. Tuchman et al., 1979; Fadeyev, 1982).

Nakata (1987) has shown that a sequence of less-massive supergiant models has a transition from regular to irregular pulsations, when the mass of the models is reduced step by step. Recently, the irregular pulsation has been investigated with respect to deterministic chaos. We concentrate in this section the bifurcation which leads irregular pulsations. It is well-known that there are universal routes from regular to irregular behaviors represented by simple mathematical models and confirmed by computer simulations and experiment measurements (Schuster, 1988). So far, there have been found two typical routes of transitions from regular to irregular pulsations in lessmassive supergiant star models: the period doubling cascade (Buchler and Kovács, 1987) and the intermittency (Aikawa, 1987: Buchler et al., 1987).

\subsection{Period Doubling Cascade}

Buchler and Kovács (1987) have shown that a model sequence for Population II Cepheids has transitions from regular to irregular pulsations through the period-doubling cascade, one of universal routes of transitions from regular to irregular behaviors in deterministic chaos. Models of the sequence have the following stellar parameters: $M=0.6 M_{\odot}, L=500 L_{\odot}$, and chemical composition: $x=0.745, z=0.005$. The effective temperature is a control parameter of the model sequence. The models with higher effective temperature have stable limit cycles. They, however, show that the sequence suffers a subharmonic bifurcation, decreasing the effective temperature of models, step by step. At this point, the limit cycle becomes unstable, but the model has a stable periodic oscillation with a period of $2^{1}$ times the period of unstable limit cycle. The sequence suffers another subharmonic bifurcation again, in the course of decrease of the effective temperature, and then has models with $2^{2}$ periods. The subharmonic bifurcation repeats indefinitely ( the period doubling cascade ) and finally leads to chaotic pulsation. 
Aikawa (1990) has demonstrated using TGRID hydro code that the period doubling cascade in one of model sequences studied by Buchler and Kovács (1987) is interrupted by the intermittency. Goupil et al. (1991) have suggested that analysis with higher dimension rather than the conventional first one-dimensional maps may reveal the nature of chaotic pulsation in this model.

\subsection{INTERMITTENCY}

Aikawa (1987) performed more luminous model sequence of less-massive supergiant stars. The model parameters are: $L=3200_{c}, T \epsilon=5300 \mathrm{~K}$, and chemical composition: $x=0.70, z=0.02$. The mass is a control parameter of the sequence. The results with LNA analysis are tabulated in Table I. The sequence shows different type of the transition from limit cycles to chaos, as shown in Fig. 4.

TABLE I

\begin{tabular}{cccccc}
\hline Model & $M / M_{\odot}$ & $P_{f}^{a}$ & $\eta_{f}^{b}$ & $P_{1}$ & $\eta_{1}$ \\
\hline $\mathrm{a}$ & 1.50 & 27.755 & 0.417 & 15.038 & -0.752 \\
$\mathrm{~b}$ & 1.46 & 28.329 & 0.421 & 15.271 & -0.786 \\
$\mathrm{c}$ & 1.45 & 28.476 & 0.422 & 15.331 & -0.795 \\
$\mathrm{~d}$ & 1.43 & 28.775 & 0.424 & 15.453 & -0.812 \\
$\mathrm{e}$ & 1.42 & 28.927 & 0.424 & 15.515 & -0.821 \\
$\mathrm{f}$ & 1.40 & 29.236 & 0.425 & 15.640 & -0.840 \\
\hline
\end{tabular}

a) The periods in units of days.

b) The growth rates are defined as $-4 \pi \omega_{i} / \omega_{r}$.

We shall make the one-dimensional return map for the hydrodynamic pulsation models. We pick up values of total pulsational kinetic energies at their maxima. During one oscillation, the quantity has two maxima at the expansion and contraction phases, and we pick up one at the expansion phase. Fig. 5 shows these return maps for chaotic pulsation in a model as well as stable limit cycles.

It is shown from Fig. 5 that in chaotic pulsation with parameters close those to stable limit cycles, nonlinear pulsation stays at a 'ghost' limit cycle for a while, but gradually obtains pulsationally kinetic energies and finally moves away from the 'ghost' limit cycle, causing an outburst. By dissipation of the kinetic energies by shock waves, the pulsation is suddenly quenched. Then, the model repeats the previous process in a similar fashion. We indentify this transition from limit cycles to chaotic oscillations in the hydrodynamic models as the type I intermittency, another universal route in simple dynamical models with dissipations (Pomeau and Manneville, 1980). 

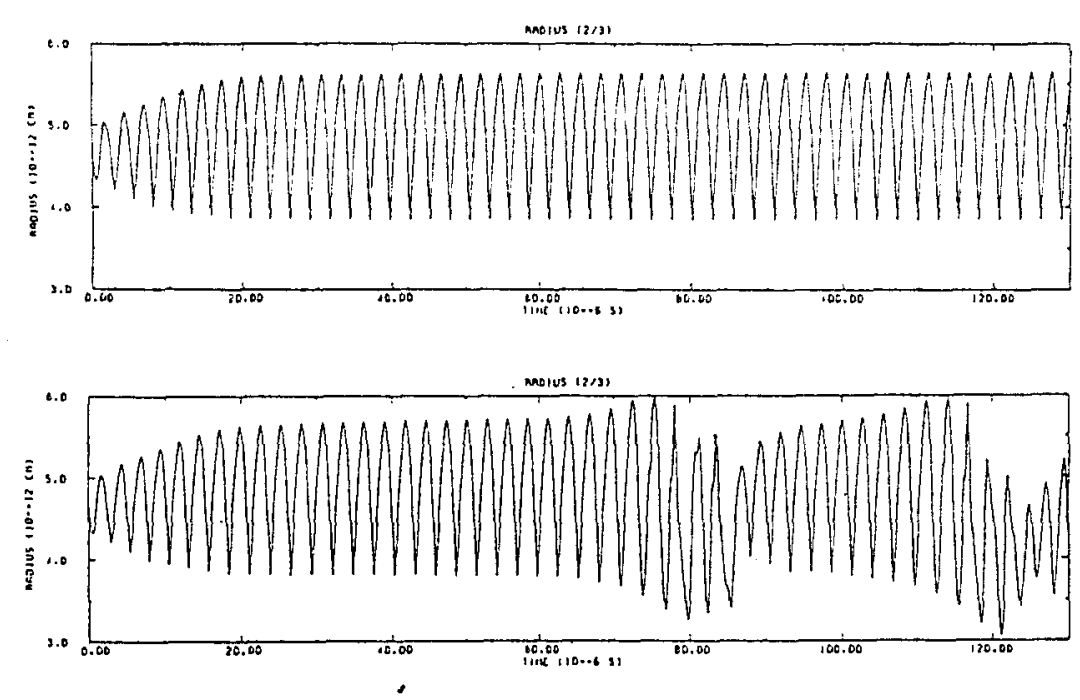

Fig. 4. Variations of the radius at the photosphere for model $\mathrm{c}$ (above) and $\mathrm{d}$ (below). It is noted that model $d$ shows irregular oscillations with outbursts at times, while model $c$ has a stable limit cycle.

Buchler et al. (1987) have demonstrated the intermittent chaos in Pop II Cepheid models.

\subsection{Dissipation Structure}

We shall discuss physical mechanisms for the transition. Aikawa (1987) has shown that the model which has stable limit cycles in the vicinity of the transition to the intermittent chaos has another unstable fixed point beyond a stable fixed point, and the transition from regular to irregular pulsation may be connected with disappearance of these fixed points (Tangent bifurcation). Aikawa (1988) shows these characteristics directly from the work integral of the nonlinear pulsations in one of models in question. It is shown that pulsation driving at the hydrogen ionization zones is strongly enhanced at amplitudes beyond the stable limit cycle. This additional driving makes the models to have another unstable fixed point.

It is suspected that the dissipation structures at the ionization zones with shock wave dissipation, as discussed in Aikawa (1988) may be also responsible for chaotic pulsations with other types of transition in less-massive supergiant models (see, Takeuti, 1987). 

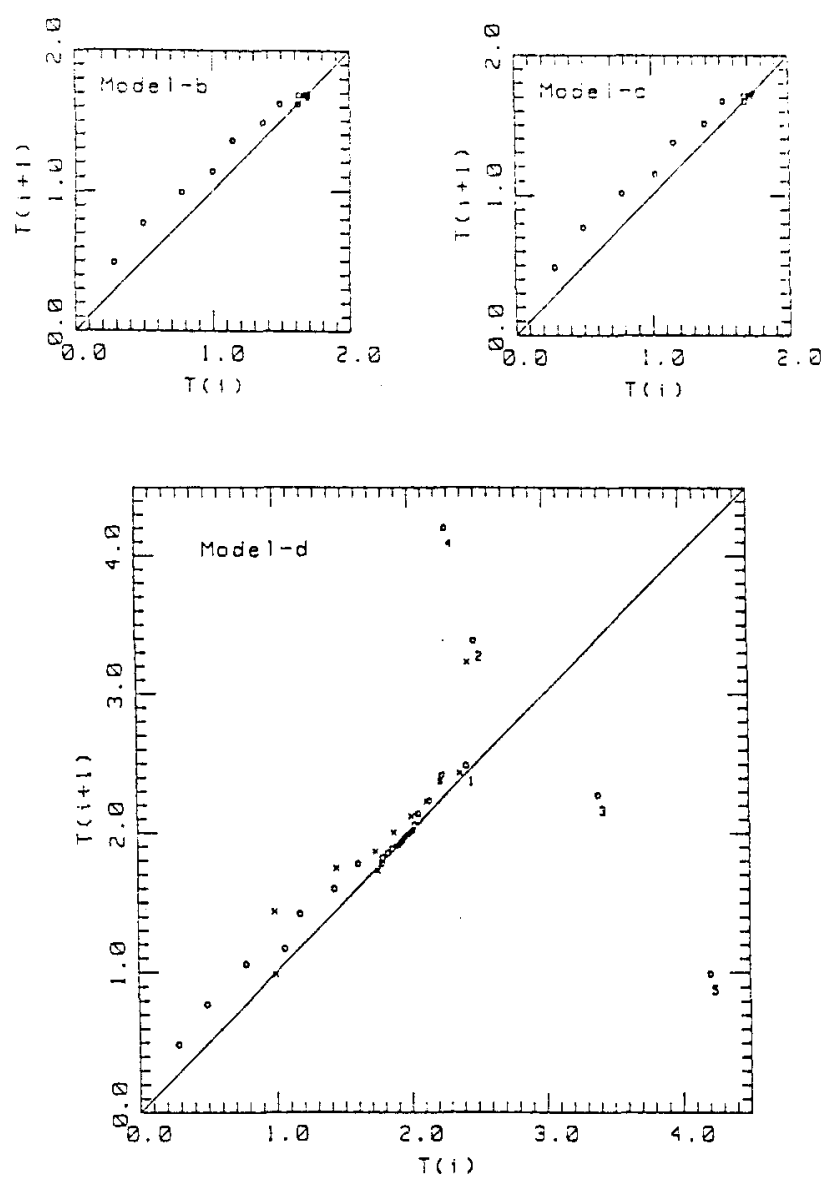

Fig. 5. The one-dimensional return maps for limit cycles (above) and irregular pulsation of model d (below). The latter model stays the bisectrix for a long time, following an outburst of large amplitudes. The oscillation is then suddenly quenched and restarts with a small amplitudes. The symbol $(O)$ and $(x)$ are for first and second cycles of this process. The data points at the outburst are numbered in chronological order.

\subsection{Dynamic Regimes in the HR Diagram}

Kovács and Buchler (1988) have demonstrated with investigation of transitions from regular to irregular pulsations of model sequences of different luminosities. The transition in lower luminosity sequences is induced by the period doubling cascade, and on the other hand, the intermittency for higher luminosity models. 


\section{Bifurcation in Pulsation Models for The Post-AGB}

\subsection{Pulsation in Yellow Supergiaxt Stars}

Pulsation in yellow supergiant stars (e.g. 89 Her and HD 161796) has the following characteristics:

(1) Irregular pulsation with time scale of about 40 days,

(2) small amplitudes about 0.2 mag., which is contrasted with irregular

variables in red giant stars,

(3) Some of them are suggested the post-asymptotic Giant Branch (postAGB) stars.

The time variations of magnitude in $89 \mathrm{Her}$ and HD161796, the prototypes of this class have been obtained by Fernie through the Automatic Photometric Telescope (APT) Service since Fernie (1983). Recently, Zsoldos and Sasselov (1991) have claimed that complicated light variation in UU Her which used to be classified as this class can be explained by superpostion of two linear oscillators with slowly modulated amplitudes. Thus, we need careful examinations for analysis of the variations.

\subsection{Linear Models}

The observed effective temperature of these yellow supergiant stars indicates that these stars are located at bluer region outside the conventional instability strip for pulsating stars in the HR diagram. Aikawa (1991) has shown that less-massive supergiant stars (e.g. $M<1 M_{\odot}$ ) with appropriate luminosities are unstable by overstable oscillations with higher overtone modes, while the modes have different properties from ordinary modes and must be related with strange modes (Gautschy and Glatzel, 1990), but the physical interpretation on the driving of the mode is unclear.

\subsection{Pulsations in Nonlinear Regime}

Nonlinear simulations are performed for models of $M=0.8 M_{\odot}$ with sequences of models with different values of the effective temperature ( $T e=$ $6000 \mathrm{~K}, 6300 \mathrm{~K}, 6600 \mathrm{~K}, 6900 \mathrm{~K}, 7200 \mathrm{~K}$ and $7500 \mathrm{~K}$ ) to cover the region of the HR diagram for $F$ type supergiant stars. The luminosity is a control parameter in this study and is changed with the range of $3500 L_{\odot}<L<$ $7000 L \odot$.

For all model sequences except for the sequence of $T e=6000 \mathrm{~K}$, models with higher luminosities show chaotic pulsation with small amplitudes, while pulsation in lower luminosity models is rather regular. It is noted that subharmonic components of the driving mode are strongly enhanced in nonlinear pulsation in chaotic regime, and time variations with much longer time scale are induced in chaotic oscillations, compared with the linear period of about 10 days.

We conclude that chaotic pulsation with small amplitudes can be gener- 


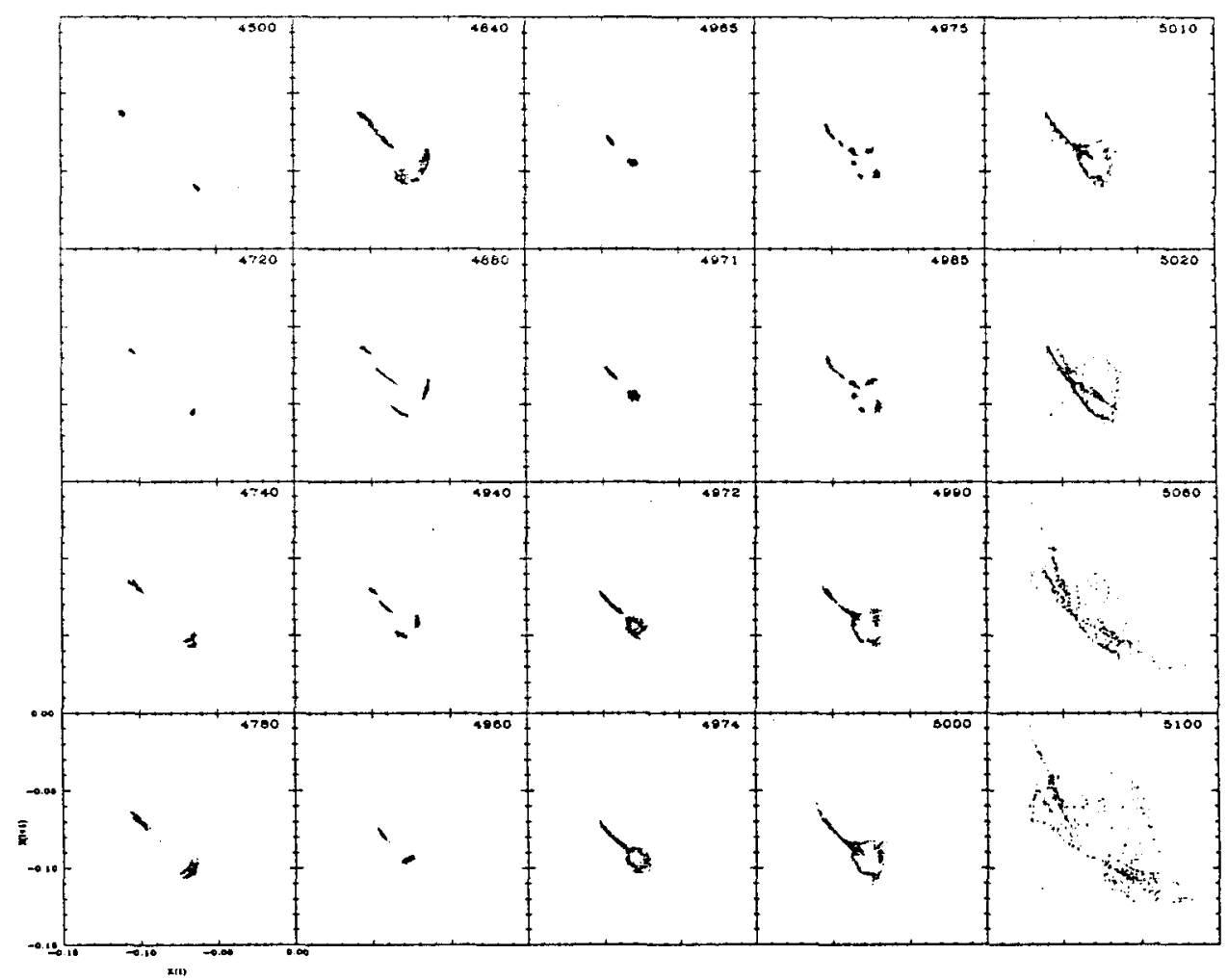

Fig. 6. The series of the one-dimensional return map for model sequence $T e=6300 \mathrm{~K}$. Only higher luminosities part is drawn and luminosities of models are labeled for each map. Data of the Poincare section are made from data on magnitude at the photosphere at the time of maximum expansion of the same place.

ated robustly in pulsation of less-massive supergiant stars located apparently at bluer region outside the conventional blue edge.

The model sequence of $T e=6300 \mathrm{~K}$ is studies in detail to find out nature of chaotic pulsation. The model sequence shows much complicated transitions from regular to chaotic pulsations, as demonstrated in Fig. 6 as a series of the first return maps for the higher luminosity part in the model sequence.

Plotting data of the Poincare section with $3 \mathrm{D}$, we can see more clearly the structure of transition, as shown in Fig. 7 and so the transition may be understood in higher dimensional mappings. The properties are often appeared in the transitions to chaos in which universal routes are interrupted by periodic oscillations and other type routes (Arneodo et al., 1983). 


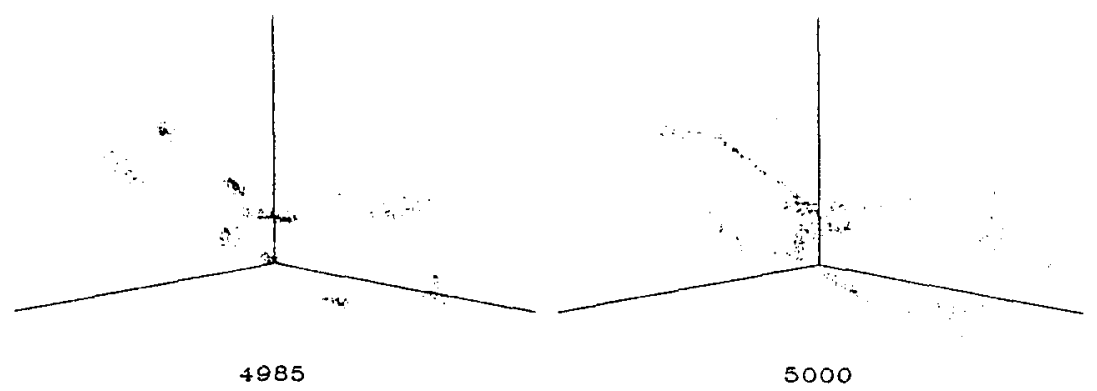

Fig. 7. 3D plots of the Poincare section data. Three successive data are plotted in three dimensional space. For model $L=4985 L_{\odot}$, we can see a periodic oscillation with 9 period, and this periodic oscillation becomes divergent in model $L=5000 L_{\odot}$.

\section{Conclusions}

Phenomena of bifurcation in non-linear pulsation in hydrodynamic models are reviewed. It is pointed out that qualitatively different behaviors in nonlinear pulsation are realized with transitions due to bifurcation in hydrodynamic models. We may summarize as follows:

(1) In weakly dissipative models (Cepheid Models), bifurcation in nonlinear pulsation is induced by modal resonances in the fundamental and first overtone pulsators. Some of them are responsible for observed properties.

(2) In strongly dissipative models (less-massive supergiant stars), there have been found two systematic routes of transition from regular to chaotic pulsations in hydrodynamic models: the period-doubling cascade and the tangential bifurcation. These transitions are induced by the dissipation structure of the ionization zones including shock waves generated in the region.

(3) Models for chaotic pulsation with small amplitudes in the post-AGB stars are proposed. There are transitions from regular to irregular pulsations with small amplitudes in a wide range of stellar parameters. Thus, the transitions are robust for generation of chaotic pulsations with small amplitudes.

\section{References}

Aikawa, T.: 1987, in Stellar Pulsation, eds. A. N. Cox, W. M. Sparks and S. G. Starrfield (New York: Springer), p. 175.

Aikawa, T.: 1987, Astrophysics and Space Science, 139, 281.

Aikawa, T.: 1988, Astrophysics and Space Science, 149, 149.

Aikawa, T.: 1990, Astrophysics and Space Science, 164, 295.

Aikawa, T.: 1991, Astrophysical Journal, 374, 700.

Antonello, E.: 1991, private communication.

Antonello, E., Poretti, E., and Reduzzi, L.: 1990, Astronomy and Astrophysics, 236, 138. Aeneodo, A., Coullet, P., Tresser, C., Libchaber, A., Maurer, J. and d'Humieres, D.: 1983, Physica, 6D, 385. 
Balona, L. A.: 1985, in Cepheids: Theory and Observations, ed. B. F. Madore (Cambridge: Cambridge Univ. Press), p. 17 .

Buchler, J. R. Goupil M. -J., and Kovács, G.: 1987, Phys. Lett., A 126, 177.

Buchler, J. R. and Kovács, G.: 1987, Astrophysical Journal, Letters to the Editor, 320, L.57.

Buchler, J. R. Moskalik, P. and Kovács, G.: 1990, Astrophysical Journal, 351, 617.

Carson, T. R. and Stothers, R. B.: 1988, Astrophysical Journal, 328, 196.

Chiosi, C.: 1990, in Confrontation between Stellar Pulsation and Evolution, eds. C. Cacciari and G. Clementini (ASP Conf. Ser. vol.11), p. 158.

Fadeyev, Y. A.: 1982, Astrophysics and Space Science, 86. 143.

Fadeyev, Y. A.: 1984, Astrophysics and Space Science, 100, 329.

Fadeyev, Y. A.: 1992, this conference.

Fernie, J. D.: 1983, Astrophysical Journal, 265, 999.

Gautschy, A. and Glatzel, W.: 1990, Monthly Notices of the RAS, 245, 597.

Goupil, M. -J., Auvergne, M. and Serre, Th.: 1991, in Applying Fractals in Astronomy, eds. A. Heck and J. M. Perdang (New York: Springer), p. 43.

Iooss, G. and Joseph, D. D.: 1980, Elementary Stability and Bifurcation Theory (New York: Springer).

Kovács, G. and Buchler, J. R.: 1988, Astrophysical Journal, 334, 971.

Moskalik, P. and Buchler, R. J.: 1990, Astrophysical Journal, 355, 590.

Moskalik, P. and Buchler, R. J.: 1991, Astrophysical Journal, 366, 300.

Nakata, M.: 1987, Astrophysics and Space Science, 132, 337.

Saio, H.: 1992, this Conference.

Schuster, H. G.: 1988, Deterministic Chaos: an Introduction (Weinheim:VCH).

Simon, N. R.: 1982, Astrophysical Journal, Letters to the Editor, 260, L87.

Simon, N. R. and Aikawa, T.: 1986, Astrophysical Journal, 304, 249.

Simon, N. R. and Davis, C.: 1983, Astrophysical Journal, 266, 787.

Simon, N. R. and Lee, A. S.: 1981, Astrophysical Journal, 248, 291.

Simon, N. R. and Schmidt, E. G.: 1976, Astrophysical Journal, 205, 162.

Takeuti, M.: 1987, Astrophysics and Space Science, 136, 129.

Takeuti, M. and Aikawa, T.: 1981, Science Rep. Tóhoku Univ., 8-th ser., 2, 106.

Takeuti, M. Uji-iye, K, and Aikawa, T.: 1983, Science Rep. Tôhoku Univ., 8-th ser., 4, 129.

Tuchman, Y., Sach, N. and Barkat, Z.: 1979, Astrophysical Journal, 234, 217.

Zsoldos, E. and Sasselov, D. S.: 1991, preprint (to appear to Astronomy and Astrophysics). 